\title{
IMAGINE: Modeling the Galactic Magnetic Field
}

\author{
Marijke Haverkorn ${ }^{1, * \mathbb{D}}$, François Boulanger ${ }^{2}$, Torsten Enßlin ${ }^{3}$, Jörg R. Hörandel ${ }^{1,4}$, \\ Tess Jaffe ${ }^{5,6}$, Jens Jasche ${ }^{7}$, Jörg P. Rachen ${ }^{1}$ and Anvar Shukurov ${ }^{8}$ \\ 1 Department of Astrophysics/IMAPP, Radboud University, P.O. Box 9010, 6500 GL Nijmegen, \\ The Netherlands; j.horandel@astro.ru.nl (J.R.H.); J.Rachen@astro.ru.nl (J.P.R.) \\ 2 Laboratoire de Physique de l'Ecole Normale Supérieure, Université PSL and CNRS, 75005 Paris, France; \\ francois.boulanger@ias.u-psud.fr \\ 3 Max Planck Institute for Astrophysics, Karl-Schwarschildstr. 1, 85741 Garching, Germany; \\ ensslin@mpa-garching.mpg.de \\ 4 Nikhef, Science Park 105, 1098 XG Amsterdam, The Netherlands \\ 5 CRESST II, NASA Goddard Space Flight Center, Greenbelt, MD 20771, USA; tess.jaffe@nasa.gov \\ 6 Department of Astronomy, University of Maryland, College Park, MD 20742, USA \\ 7 The Oskar Klein Centre, Department of Physics, Stockholm University, AlbaNova University Centre, \\ SE 10691 Stockholm, Sweden; jens.jasche@fysik.su.se \\ 8 School of Mathematics, Statistics and Physics, Newcastle University, Newcastle Upon Tyne NE1 7RU, UK; \\ anvar.shukurov@newcastle.ac.uk \\ * Correspondence: m.haverkorn@astro.ru.nl
}

Received: 5 September 2018; Accepted: 17 December 2018; Published: 14 January 2019

\begin{abstract}
The IMAGINE Consortium aims to bring modeling of the magnetic field of the Milky Way to the next level by using Bayesian inference. IMAGINE includes an open-source modular software pipeline that optimizes parameters in a user-defined galactic magnetic field model against various selected observational datasets. Bayesian priors can be added as external probabilistic constraints of the model parameters. These conference proceedings describe the science goals of the IMAGINE consortium, the software pipeline and its inputs, namely observational data sets, galactic magnetic field models, and Bayesian priors.
\end{abstract}

Keywords: galactic magnetic fields; interstellar medium; theory of cosmic magnetism; cosmic rays

\section{Introduction}

The magnetic field of the Milky Way is an invisible agent controlling many physical processes, such as carving gas clouds into filaments, delaying star formation, accelerating and guiding cosmic rays, shaping supernova remnants, and puffing up the galactic halo. The energy density contained in these magnetic fields is comparable to that in stars, in cosmic rays, and in the turbulent gas, causing constant interaction with (and feedback from) all these components [1]. Therefore, understanding the Galactic ecosystem is a major scientific goal for studies of the magnetic field in the Milky Way. In addition, the shape and strength of magnetic fields in galaxies provides clues as to the origin and the evolution of the galactic magnetic field, which in turn can constrain our ideas of formation of galaxies and large-scale structure in the early universe. Lastly, the galactic magnetic field can also be a hindrance to studies wanting to investigate what lies beyond: It muddles our view of sources of ultra-high energy cosmic rays (UHECRs) and of extragalactic magnetic fields, it causes small-scale polarized synchrotron radiation that can mimic the signature of the $21 \mathrm{~cm}$ line of neutral hydrogen in the early universe's dark ages, and produces dust polarization that hampers cosmic microwave background polarization measurements. In summary, the magnetic field of the Milky Way is important for a broad range of research areas, whether it will be used to study it or subtract it. 
Observational and theoretical knowledge of magnetic fields in the Milky Way still give us a sketchy image [2,3]. The idea that a magnetic dynamo has amplified and maintains the Galactic magnetic field is well-established. However, it is far from clear what the most important driving agents of this dynamo are (e.g., turbulence or cosmic-ray buoyancy) [4]. Large-scale mean-field dynamo models can be described analytically as a combination of modes, some of which are more likely to be excited than others, in disk galaxies. The small-scale magnetic field structure is often modeled as Gaussian turbulence, which is too simplified as magnetic turbulence is highly anisotropic and intermittent. There are a large number of indirect observational tracers for magnetic fields in the galaxy: These probe either the strength or direction of the field, either the component parallel to the line of sight, or the component in the plane of the sky. In addition, these tracers depend on the conditions in the probed region, making reconstruction of the total field strength and direction at a certain location all but impossible.

Much research has been done in the past decades towards trying to construct a galactic magnetic field model that is consistent with observational tracers [5]. However, these studies are very heterogeneous, in the sense that they use different parameterizations of the galactic magnetic field and a different subset of observational tracers. This makes it impossible to quantitatively assess their quality and to intercompare them. The Interstellar MAGnetic field INference Engine (IMAGINE) research project aims to take the next step in Galactic magnetic field modeling, by including all possible observational tracers and using Bayesian inference techniques; allowing the inclusion of prior (observational and theoretical) knowledge about galactic magnetism, and quantifiable quality assessment of different models.

This proceedings contribution briefly discusses the aims and methods of the IMAGINE consortium. A much more elaborate discussion about the content and science goals of IMAGINE can be found in the IMAGINE white paper [5].

\section{Introducing IMAGINE}

\subsection{What is IMAGINE?}

IMAGINE is both a consortium and a software package. The consortium is an international, broad, and open group of researchers, who are joined by an interest in galactic magnetic fields. The goal of the consortium is to coordinate and facilitate the development and improvement of models for the magnetic field of the Milky Way, with the broader goal of gaining more insight into a wide variety of science questions that are influenced by galactic magnetic fields in various ways. More information about the project can be found at: https://astro.ru.nl/imagine/.

The means of achieving this is in the development and use of the IMAGINE software, which models the galactic magnetic field using Bayesian inference and all possible observational inputs and prior knowledge of the Galactic magnetic field. The general set-up of the project is laid out in the mind map in Figure 1, which we will describe in the remainder of this paper. The basic IMAGINE pipeline is written and tested [6], see Section 3. 


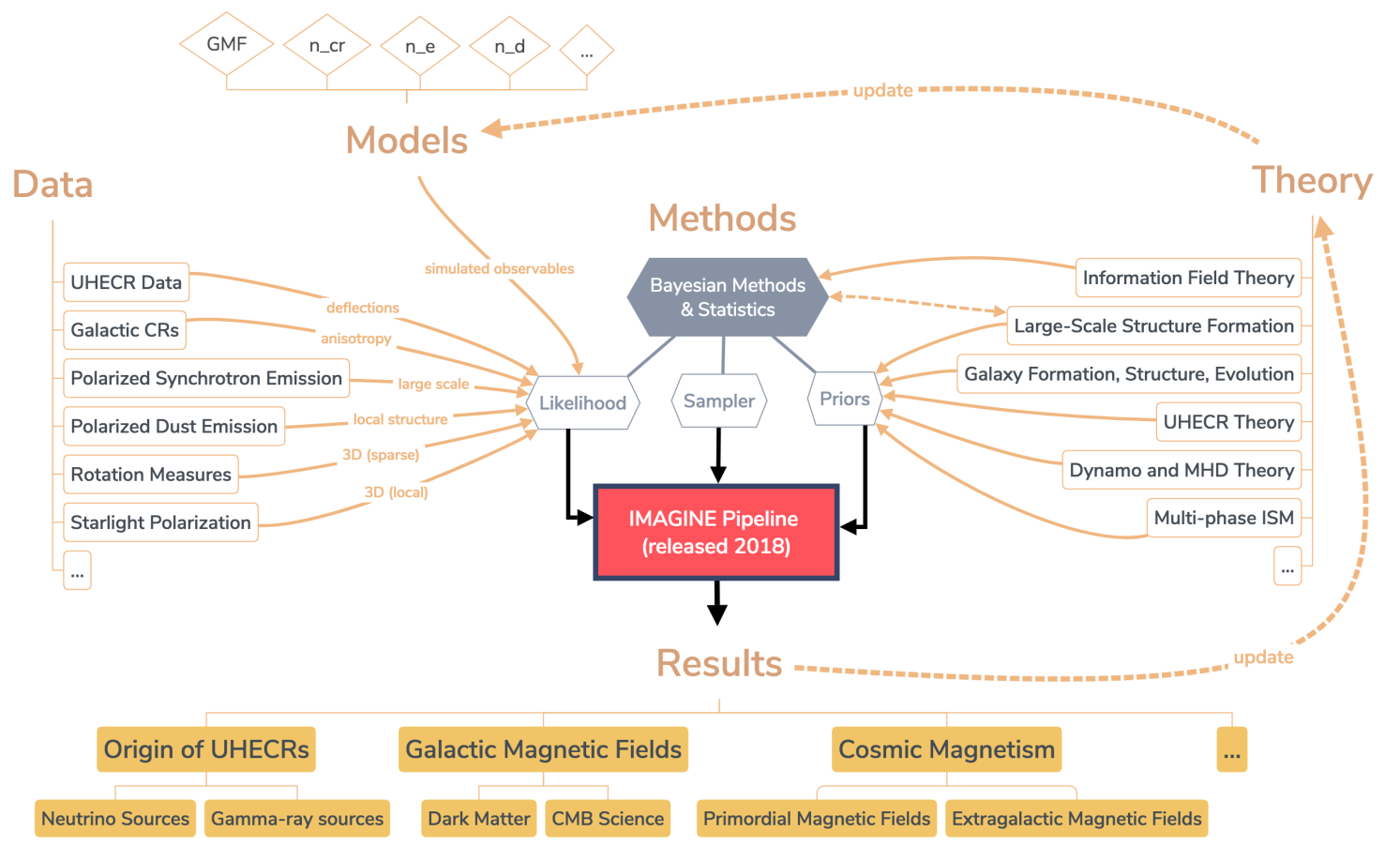

Figure 1. A mind map of the IMAGINE project [5].

\subsection{Scientific Aims of IMAGINE}

Knowledge of the strength and structure of the galactic magnetic field is indispensable for studies of the Milky Way's interstellar medium and cosmic rays. It is a valuable input for dynamo theories and studies of galactic and extragalactic magnetism. In addition, the magnetic field of the Milky Way is a foreground, influencing various extragalactic studies such as UHECR sources, cosmic microwave background polarization, or the Epoch of Reionization.

The main goal of the IMAGINE Consortium is in the modeling of the galactic magnetic field. Major scientific questions that can be addressed with sufficiently reliable knowledge of the galactic magnetic field are the following:

What is the role of the galactic magnetic field in the interstellar medium?

It is widely known that galactic magnetic fields are dynamically important, and that their energy density is comparable to other components in the Milky Way [7]. Recently, the close coupling between these components has become clear in detail: Hydrogen filaments are aligned in magnetic fields in dust, as probed by starlight polarization [8]; by polarized dust emission [9]; and by Faraday rotation [10,11]. State of the art observational data sets, such as Planck polarization maps [12], all-sky Faraday rotation measure maps [13], and many $\mathrm{HI}$ and (polarized) synchrotron studies with current and next-generation instruments, necessitate detailed knowledge on the turbulent components of the galactic magnetic field to ensure progress.

How are galactic cosmic rays accelerated and propagated in the galactic magnetic field?

Electrons, positrons, and ions are accelerated under the influence of small-scale magnetic fields to cosmic rays. These cosmic rays propagate through the galaxy mostly along field lines, but also by diffusion and advection. They interact with the interstellar environment through, for example, spallation or gas heating; understanding these processes, and the creation and propagation of galactic cosmic rays, therefore needs accurate knowledge about the local magnetic field strength and structure. 
How are magnetic fields in galaxies amplified and maintained?

There are a number of physical processes that can create very weak magnetic fields in the early universe. These fields are then amplified by a magnetic dynamo mechanism to the microGauss strengths we observe in current galaxies. How that magnetic dynamo works in detail, and what drives it, are as yet open questions. Various dynamo models give different predictions for symmetries in the large-scale structure of galactic magnetic fields. Hence, a good model of the galactic magnetic field can constrain these theories and shed light on the origin and evolution of galactic magnetism.

What are the sources of ultra-high energy cosmic rays?

UHECRs are extragalactic cosmic rays with energies exceeding $E \sim 10^{18} \mathrm{eV}$. Various cosmic sources could possibly accelerate charged particles to these extreme energies, such as compact transients connected to star forming activity in galaxies, AGN, radio galaxies, or galaxy clusters, but the relative contribution of these sources is still unknown. UHECRs propagating from their sources through the magnetized intergalactic and interstellar medium to detectors at Earth are deflected by the intergalactic and (in many directions dominant) galactic magnetic fields. The deflections in these magnetic fields currently preclude us from following these particles back to their sources. A sufficiently reliable model of the (large-scale) magnetic field of the galaxy would enable us to determine the sources of the UHECRs.

Apart from these science questions, knowledge of the magnetic field and non-thermal components of the Milky Way can be useful more tangentially in the study of galaxy formation. One of the most stringent problems in galaxy formation studies is the "missing satellite problem", i.e., the problem that numerical simulations predict many more low-mass satellite galaxies than are observed. This discrepancy can possibly be attributed to the underestimation of feedback processes by stellar winds, supernova remnants, and active galactic nuclei in these simulations. This feedback could drive gas out of galaxies, or preclude new gas from accreting onto the galaxies. Studies of the non-thermal components of the Milky Way will teach us about these feedback processes at low star formation rates.

Finally, a trustworthy model of the galactic magnetic field will allow us to properly subtract the (polarized) radiation fields in the galaxy that arise due to this magnetic field, to reveal the extragalactic sky in more detail. We will be able to better model galactic polarized dust emission to benefit detection of polarized cosmic microwave background B-modes, to model polarized galactic synchrotron emission to aid detection of HI fluctuations from the Epoch of Reionization, or to study magnetic fields in the cosmic web.

\section{The IMAGINE Software Package}

The IMAGINE software is a modular open source framework for doing inference on generic parametric models of the galaxy [6]. IMAGINE is built in PYTHON, connecting various individual components and external libraries such as NIFTy 3 [14], Hammurabi [15] and PyMultiNest [16]. Required computational resources depend on usage: a single realization of various models can be computed on a single workstation, whereas on the order of $10 \mathrm{kCPUh}$ would be needed for a full likelihood exploration of a four-dimensional parameter space. Figure 2 shows its general outline. The software is publicly available at https://gitlab.mpcdf.mpg.de/ift/IMAGINE.

Within the IMAGINE pipeline, a magnetic field configuration is generated, according to a user-defined model (see Section 5). Using this magnetic field configuration, combined with other necessary input models such as thermal electron density, cosmic ray electron density, and dust distributions, all-sky maps of observables are generated, using the Hammurabi software package [15]. The generated observables can be synchrotron intensity, synchrotron polarized intensity, polarized dust emission, or rotation measure, depending on the available data. Comparison of the generated data with observational maps (Section 4) results in likelihood evaluations for the magnetic field parameters 
(which are saved in a repository), after which a sampler selects a new set of parameters in the magnetic field model, using information from priors (Section 6) if available.

This framework ensures significant improvement of this project over earlier galactic magnetic field modeling in the literature, in multiple ways:

- Priors: any knowledge that we already have about galactic magnetic fields can be included in the modeling;

- Addition of observational tracers: the modular set-up of IMAGINE enables inclusion of different and heterogeneous data sets in a consistent way;

- Evidence: the Bayesian evidence component in IMAGINE allows quantitative comparison of various galactic magnetic field models in the literature. Apart from comparison of various models using the same data by the same authors, comparison of models in different publications using different data sets is only attempted by the Planck collaboration [17]; quantitative comparison is currently completely lacking.

A detailed description of the software framework and early tests can be found in [6].

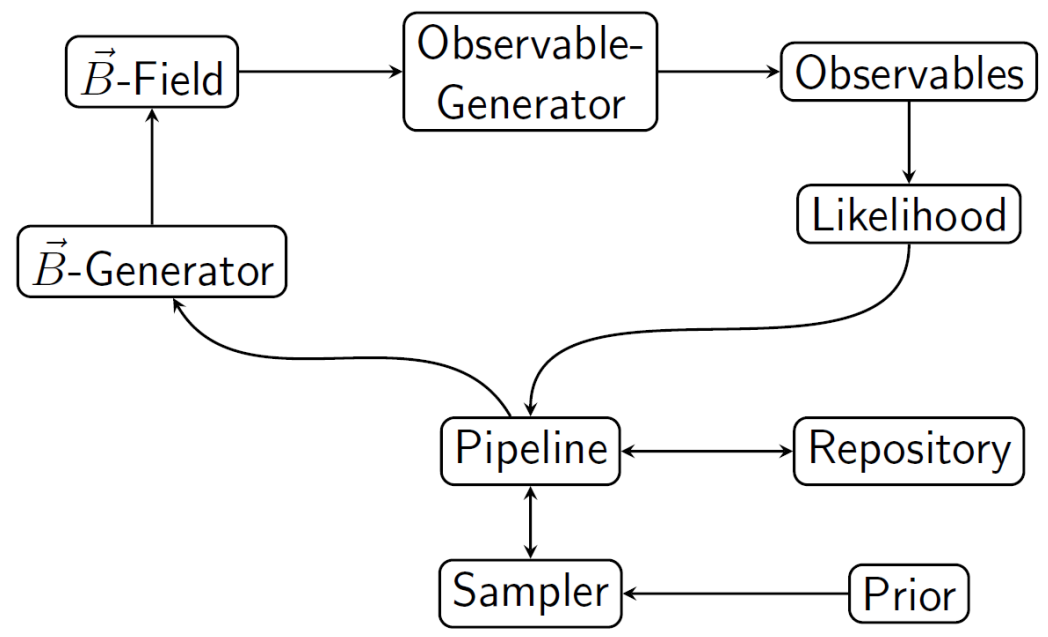

Figure 2. The structure of the IMAGINE project: Generating models, comparison to data, and sampling parameter space [5].

\section{Observational Tracers}

The ambitious goal of IMAGINE is to eventually use all possible observational tracers. This includes, first of all, the 'traditional' observational tracers, (i.e., tracers that are currently used for model fitting of the galactic magnetic field; see $[3,18])$. These are:

- (Polarized) synchrotron emission. Synchrotron emission traces the magnetic field component perpendicular to the line of sight, integrated over the line of sight. Its polarization can be used as a measure of the ratio of the turbulent to the uniform magnetic field components.

- Faraday Rotation of polarized radio emission from pulsars. Pulsar rotation measures (RMs) give information on the magnetic field component parallel to the line of sight $B_{\|}$, weighted by the electron density $n_{e}$, and integrated along the line of sight $s: \operatorname{RM} \propto \int n_{e} B_{\|} d s$. Although these data have the great advantage that they probe the magnetic field in the 3D volume of the galaxy (instead of most other tracers that integrate over pathlengths through the entire galaxy), pulsar RMs are fairly scarce and are mostly concentrated near the Galactic plane.

- Rotation measures from extragalactic point sources. Mostly thanks to the RMs from the all-Northern-sky NVSS survey [19], the RM grid from extragalactic sources now contains $\sim 40,000$ sources across the whole sky, from which a Faraday rotation map of the sky can be constructed [13]. 
- Thermal emission from dust is partially polarized, due to the influence of magnetic fields. All-sky dust polarization maps (from e.g., the Planck satellite) combined with a dust model, give an independent measure of the averaged magnetic field component perpendicular to the line of sight.

A fifth much used and versatile observational tool to detect in-situ interstellar magnetic fields, Zeeman splitting, is not included here. This is because Zeeman splitting probes the cold neutral gas in dense clouds (e.g., in star forming regions), or gas in very specific circumstances and environments (such as masers). At increasing gas densities, the magnetic fields in these environments are believed to be significantly altered with respect to the global field structure in the diffuse medium ([20], however, also see [21]).

In addition to these tracers, there are a number of observational tracers that have, so far, been used little (if at all) to probe the large-scale magnetic field due to great complexity or uncertainties. However, improved technological capabilities are making it worthwhile to start thinking about these tracers. They are:

- Starlight polarization: Optical and near-infrared starlight gets partially polarized by elongated dust grains, rotating aligned with an interstellar magnetic field. Small fields of view have been used to constrain galactic magnetic field models [22]. Future all-sky optical polarimetric surveys [23] combined with GAIA distances and reliable dust models will be included into IMAGINE. The short lines of sight to the stars will make this data set very complementary to the traditional tracers that integrate along the line of sight through the entire galaxy.

- Galactic cosmic ray distribution and spectral dependence. Small-scale anisotropies in galactic TeV cosmic ray arrival directions, recently discovered by several experiments [24,25], can (in principle) be predicted in the galaxy model generated in IMAGINE, which can provide additional constraints to the model parameters. In addition, spectral information in the synchrotron radiation, depending on CR electron spectra (see e.g., [26,27]), can be used as well.

- UHECR arrival directions: UHECRs are deflected by the galactic magnetic field, for the highest measured energies typically by a few degrees to a few tens of degrees (depending on their rigidity and propagation path). A Bayesian optimization of the source distribution(s) of UHECRs as well as their deflections in intergalactic space and in the galaxy against their arrival directions at Earth can be used to constrain possible galactic magnetic field configurations.

- Faraday tomography: in principle, Faraday tomography maps of the IMAGINE galaxy model could be compared to observed Faraday tomography maps. Faraday tomography at low (LOFAR, MWA) frequencies reveals more about discrete structures in the local neighborhood and therefore may not be ideal. However, at higher $(\mathrm{GHz})$ frequencies, Faraday depth spectra likely better represent the global galactic magnetic field, and, as such, can be used to constrain model parameters.

\section{Galactic Magnetic Field Models}

A large number of parameterized, heuristic models of the galactic magnetic field exist, which are heterogeneous in many different ways. These models fit the galactic disk only (e.g., [28]) or fit both disk and halo magnetic fields. They include different magnetic field components, such as regular and turbulent magnetic fields, anisotropic turbulent magnetic fields (e.g., [29]), or vertical magnetic fields (e.g., [30,31]). They can use either simple Gaussian slabs to estimate the underlying cosmic-ray density, or complex models like GALPROP [32] based on observational data [33]. Also, they are based on various observational data sets, such as (a subset of) extragalactic source rotation measures, pulsar rotation measures (e.g., [34]), synchrotron intensity, synchrotron polarization (e.g., [35]), thermal dust emission (e.g., [36]), and thermal dust polarization (e.g., [37]) (see [2] for a review).

Using IMAGINE, it becomes relatively straightforward to quantify how well each of these models fit the data, by computing the Bayesian evidence. This will take into account possible varying 
observational data and varying numbers of free parameters. Also, all models can be compared by fitting against the same observational data.

As a next step, we intend to include galactic magnetic field models based on physical coherent field configurations (e.g., [38]) or including non-Gaussian turbulent fields [39]. Eventually, the goals include exploring non-parametric modeling of the galactic magnetic field, in which the parameters are treated as fields optimized under certain prior constraints (see e.g., [40]) .

\section{Bayesian Priors}

Any existing knowledge of the galactic magnetic field can be coded as a Bayesian prior, and used as a constraint in the model fitting.

Prior information not used in the heuristic galactic magnetic field models, described above, are the equations describing the physical processes underlying the magnetic field configuration, i.e., the dynamo equations. Any magnetic field can be described as a superposition of various dynamo modes, which are eigenfunctions of the mean-field dynamo equation. As it is much more likely to observe a growing dynamo mode than a decaying one, the probabilities of these eigenvalues of the dynamo modes can be used as prior information in the modeling.

In addition, any constraints on the galactic magnetic field, known from previous (unrelated) observational data, can be used as a prior, such as information on the magnetic field in the local Solar neighborhood (e.g., [41]) or about large-scale field reversals in the extended Solar neighborhood (e.g., [42]). Magnetic fields have been measured in dozens of nearby spiral galaxies [18], which together provide rich constraints on probabilities of various galactic magnetic field configurations.

More possible general priors are based on the cosmic evolution of galactic magnetic fields, by combining galactic magnetic field measurements in the early universe [43] with cosmological simulations of galaxy formation.

Finally, priors can be constructed, following the original idea, behind the IMAGINE consortium: combining knowledge on UHECRs and galactic magnetism. The sources of UHECRs are still not known, but the many observations have narrowed down the possibilities to a few physically plausible source categories. These can be assigned probabilities, after which observed UHECR arrival directions at Earth can be used to constrain galactic magnetic field models.

\section{Conclusions}

IMAGINE is a global effort to take modeling of galactic magnetic fields to the next level. There are a multitude of scientific questions in many different fields of astrophysics that could benefit from the usage of the IMAGINE software. The current IMAGINE consortium is addressing some of these, but there is still a lot of uncharted terrain. The consortium encourages usage of the software pipeline, and welcomes new members.

Author Contributions: Writing-original draft preparation, M.H.; writing—review and editing, all; project development and administration, all.

Funding: M.H. acknowledges funding from the European Research Council (ERC) under the European Union's Horizon 2020 research and innovation programme (grant agreement No 772663). T.E. acknowledges partly support by the DFG Cluster of Excellence "Origin and Structure of the Universe" and by the DFG Research Unit 1254 "Magnetisation of Interstellar and Intergalactic Media - The Prospects of Low-Frequency Radio Observations". A.S. thanks STFC (ST/N00900/1) and The Leverhulme Trust (RPG-2014-427) for funding.

Acknowledgments: We thank the International Space Science Institute in Bern, Switzerland, and the Lorentz Center in Leiden, the Netherlands, for the hospitality and financial support which has led to the founding of the IMAGINE Consortium. We are grateful to the anonymous referees for their constructive comments.

Conflicts of Interest: The authors declare no conflict of interest.

\section{References}

1. Ferrière, K.M. The interstellar environment of our galaxy. Rev. Mod. Phys. 2001, 73, 1031-1066. [CrossRef] 
2. Haverkorn, M. Magnetic Fields in the Milky Way. In Magnetic Fields in Diffuse Media; Lazarian, A., de Gouveia Dal Pino, E.M., Melioli, C., Eds.; Astrophysics and Space Science Library; Springer: Berlin/Heidelberg, Germany, 2015; Volume 407, p. 483. [CrossRef]

3. Han, J.L. Observing Interstellar and Intergalactic Magnetic Fields. Annu. Rev. Astron. Astrophys. 2017, 55, 111-157. [CrossRef]

4. Shukurov, A. On the origin of galactic magnetic fields. Astrophys. Space Sci. 2002, 281, 285-288. [CrossRef]

5. Boulanger, F.; Enßlin, T.; Fletcher, A.; Girichides, P.; Hackstein, S.; Haverkorn, M.; Hörandel, J.R.; Jaffe, T.; Jasche, J.; Kachelrieß, M.; et al. IMAGINE: A comprehensive view of the interstellar medium, Galactic magnetic fields and cosmic rays. J. Cosmol. Astropart. Phys. 2018, 8, 049. [CrossRef]

6. Steininger, T.; Enßlin, T.A.; Greiner, M.; Jaffe, T.; van der Velden, E.; Wang, J.; Haverkorn, M.; Hörandel, J.R.; Jasche, J.; Rachen, J.P. Inferring Galactic magnetic field model parameters using IMAGINE-An Interstellar MAGnetic field INference Engine. arXiv 2018, arXiv:1801.0434.

7. Heiles, C.; Haverkorn, M. Magnetic Fields in the Multiphase Interstellar Medium. Space Sci. Rev. 2012, 166, 293-305. [CrossRef]

8. McClure-Griffiths, N.M.; Dickey, J.M.; Gaensler, B.M.; Green, A.J.; Haverkorn, M.; Strasser, S. The Southern Galactic Plane Survey: H I Observations and Analysis. Astrophys. J. Suppl. 2005, 158, 178-187. [CrossRef]

9. Clark, S.E.; Peek, J.E.G.; Putman, M.E. Magnetically Aligned H I Fibers and the Rolling Hough Transform. Astrophys. J. 2014, 789, 82. [CrossRef]

10. Kalberla, P.M.W.; Kerp, J. Anisotropies in the HI gas distribution toward 3C 196. Astron. Astrophys. 2016, 595, A37. [CrossRef]

11. Kalberla, P.M.W.; Kerp, J.; Haud, U.; Haverkorn, M. H I anisotropies associated with radio-polarimetric filaments. Steep power spectra associated with cold gas. Astron. Astrophys. 2017, 607, A15. [CrossRef]

12. Planck Collaboration; Adam, R.; Ade, P.A.R.; Aghanim, N.; Arnaud, M.; Ashdown, M.; Aumont, J.; Baccigalupi, C.; Banday, A.J.; Barreiro, R.B.; et al. Planck 2015 results. VIII. High Frequency Instrument data processing: Calibration and maps. Astron. Astrophys. 2016, 594, A8. [CrossRef]

13. Oppermann, N.; Junklewitz, H.; Robbers, G.; Bell, M.R.; Enßlin, T.A.; Bonafede, A.; Braun, R.; Brown, J.C.; Clarke, T.E.; Feain, I.J.; et al. An improved map of the Galactic Faraday sky. Astron. Astrophys. 2012, 542, A93. [CrossRef]

14. Steininger, T.; Dixit, J.; Frank, P.; Greiner, M.; Hutschenreuter, S.; Knollmüller, J.; Leike, R.; Porqueres, N.; Pumpe, D.; Reinecke, M.; et al. NIFTy 3-Numerical Information Field Theory-A Python framework for multicomponent signal inference on HPC clusters. arXiv 2017, arXiv:1708.01073.

15. Waelkens, A.; Jaffe, T.; Reinecke, M.; Kitaura, F.S.; Enßlin, T.A. Simulating polarized Galactic synchrotron emission at all frequencies. The Hammurabi code. Astron. Astrophys. 2009, 495, 697-706. [CrossRef]

16. Buchner, J.; Georgakakis, A.; Nandra, K.; Hsu, L.; Rangel, C.; Brightman, M.; Merloni, A.; Salvato, M.; Donley, J.; Kocevski, D. X-ray spectral modelling of the AGN obscuring region in the CDFS: Bayesian model selection and catalogue. Astron. Astrophys. 2014, 564, A125. [CrossRef]

17. Planck Collaboration.; Adam, R.; Ade, P.A.R.; Alves, M.I.R.; Ashdown, M.; Aumont, J.; Baccigalupi, C.; Banday, A.J.; Barreiro, R.B.; Bartolo, N.; et al. Planck intermediate results. XLII. Large-scale Galactic magnetic fields. Astron. Astrophys. 2016, 596, A103. [CrossRef]

18. Beck, R. Magnetic fields in spiral galaxies. Astron. Astrophys. Rev. 2015, 24, 4. [CrossRef]

19. Taylor, A.R.; Stil, J.M.; Sunstrum, C. A Rotation Measure Image of the Sky. Astrophys. J. 2009, 702, 1230-1236. [CrossRef]

20. Crutcher, R.M. Magnetic Fields in Molecular Clouds. Annu. Rev. Astron. Astrophys. 2012, 50, 29-63. [CrossRef]

21. Han, J.L.; Zhang, J.S. The Galactic distribution of magnetic fields in molecular clouds and HII regions. Astron. Astrophys. 2007, 464, 609-614. [CrossRef]

22. Pavel, M.D.; Clemens, D.P. H II Region Driven Galactic Bubbles and Their Relationship to the Galactic Magnetic Field. Astrophys. J. 2012, 760, 150. [CrossRef]

23. Magalhães, A.M. SOUTH POL: Revealing the Polarized Southern Sky; Revista Mexicana de Astronomia y Astrofisica Conference Series; American Astronomical Society: Washington, DC, USA, 2014; Volume 44, p. 209.

24. Amenomori, M.; Bi, X.J.; Chen, D.; Cui, S.W.; Danzengluobu.; Ding, L.K.; Ding, X.H.; Fan, C.; Feng, C.F.; Feng, Z.; et al. On Temporal Variations of the Multi-TeV Cosmic Ray Anisotropy Using the Tibet III Air Shower Array. Astrophys. J. 2010, 711, 119-124. [CrossRef] 
25. Aartsen, M.G.; Abraham, K.; Ackermann, M.; Adams, J.; Aguilar, J.A.; Ahlers, M.; Ahrens, M.; Altmann, D.; Anderson, T.; Ansseau, I.; et al. Anisotropy in Cosmic-Ray Arrival Directions in the Southern Hemisphere Based on Six Years of Data from the IceCube Detector. Astrophys. J. 2016, 826, 220. [CrossRef]

26. Orlando, E.; Strong, A. Galactic synchrotron emission with cosmic ray propagation models. Mon. Not. R. Astron. Soc. 2013, 436, 2127-2142. [CrossRef]

27. Orlando, E. Imprints of cosmic rays in multifrequency observations of the interstellar emission. Mon. Not. R. Astron. Soc. 2018, 475, 2724-2742. [CrossRef]

28. Van Eck, C.L.; Brown, J.C.; Stil, J.M.; Rae, K.; Mao, S.A.; Gaensler, B.M.; Shukurov, A.; Taylor, A.R.; Haverkorn, M.; Kronberg, P.P.; McClure-Griffiths, N.M. Modeling the Magnetic Field in the Galactic Disk Using New Rotation Measure Observations from the Very Large Array. Astrophys. J. 2011, 728, 97. [CrossRef]

29. Jaffe, T. R.; Leahy, J. P.; Banday, A. J.; Leach, S. M.; Lowe, S. R.; Wilkinson, A. Modelling the Galactic magnetic field on the plane in two dimensions. Mon. Not. R. Astron. Soc. 2010, 401, 1013. [CrossRef]

30. Jansson, R.; Farrar, G.R. A New Model of the Galactic Magnetic Field. Astrophys. J. 2012, 757, 14. [CrossRef]

31. Jansson, R.; Farrar, G.R. The Galactic Magnetic Field. Astrophys. J. Lett. 2012, 761, L11. [CrossRef]

32. Strong, A.W.; Moskalenko, I.V. Propagation of Cosmic-Ray Nucleons in the Galaxy. Astrophys. J. 1998, 509, 212-228. [CrossRef]

33. Jaffe, T.R.; Banday, A.J.; Leahy, J.P.; Leach, S.; Strong, A.W. Connecting synchrotron, cosmic rays and magnetic fields in the plane of the Galaxy. Mon. Not. R. Astron. Soc. 2011, 416, 1152-1162. [CrossRef]

34. Men, H.; Ferrière, K.; Han, J.L. Observational constraints on models for the interstellar magnetic field in the Galactic disk. Astron. Astrophys. 2008, 486, 819-828. [CrossRef]

35. Sun, X.H.; Reich, W.; Waelkens, A.; Enßlin, T.A. Radio observational constraints on Galactic 3D-emission models. Astron. Astrophys. 2008, 477, 573-592. [CrossRef]

36. Fauvet, L.; Macías-Pérez, J. F.; Aumont, J.; Désert, F. X. ; Jaffe, T. R.;Banday, A. J. ; Tristram, M. ; Waelkens, A. H. ; Santos, D. Joint 3D modelling of the polarized Galactic synchrotron and thermal dust foreground diffuse emission. Astron. Astrophys. 2011, 526, A145. [CrossRef]

37. Jaffe, T.R.; Ferrière, K.M.; Banday, A.J.; Strong, A.W.; Orlando, E.; Macías-Pérez, J.F.; Fauvet, L.; Combet, C.; Falgarone, E. Comparing polarized synchrotron and thermal dust emission in the Galactic plane. Mon. Not. R. Astron. Soc. 2013, 431, 683-694. [CrossRef]

38. Ferrière, K.; Terral, P. Analytical models of X-shape magnetic fields in galactic halos. Astron. Astrophys. 2014, 561, A100. [CrossRef]

39. Shukurov, A.; Snodin, A.P.; Seta, A.; Bushby, P.J.; Wood, T.S. Cosmic Rays in Intermittent Magnetic Fields. Astrophys. J. Lett. 2017, 839, L16. [CrossRef]

40. Enßlin, T.A.; Frommert, M.; Kitaura, F.S. Information field theory for cosmological perturbation reconstruction and nonlinear signal analysis. Phys. Rev. D 2009, 80, 105005. [CrossRef]

41. Alves, M.I.R.; Boulanger, F.; Ferrière, K.; Montier, L. The Local Bubble: A magnetic veil to our Galaxy. Astron. Astrophys. 2018, 611, L5. [CrossRef]

42. Simard-Normandin, M.; Kronberg, P.P. New large-scale magnetic features of the Milky Way. Nature 1979, 279, 115-118. [CrossRef]

43. Farnes, J.S.; O'Sullivan, S.P.; Corrigan, M.E.; Gaensler, B.M. Faraday Rotation from Magnesium II Absorbers toward Polarized Background Radio Sources. Astrophys. J. 2014, 795, 63. [CrossRef]

(C) 2019 by the authors. Licensee MDPI, Basel, Switzerland. This article is an open access article distributed under the terms and conditions of the Creative Commons Attribution (CC BY) license (http:/ / creativecommons.org/licenses/by/4.0/). 